\title{
Animal telemetry: Tagging effects
}

\author{
Trevor McIntyre
}

Mammal Research Institute, Department of Zoology and Entomology, University of Pretoria, Hatfield 0028, South Africa.

E-mail: tmcintyre@zoology.up.ac.za

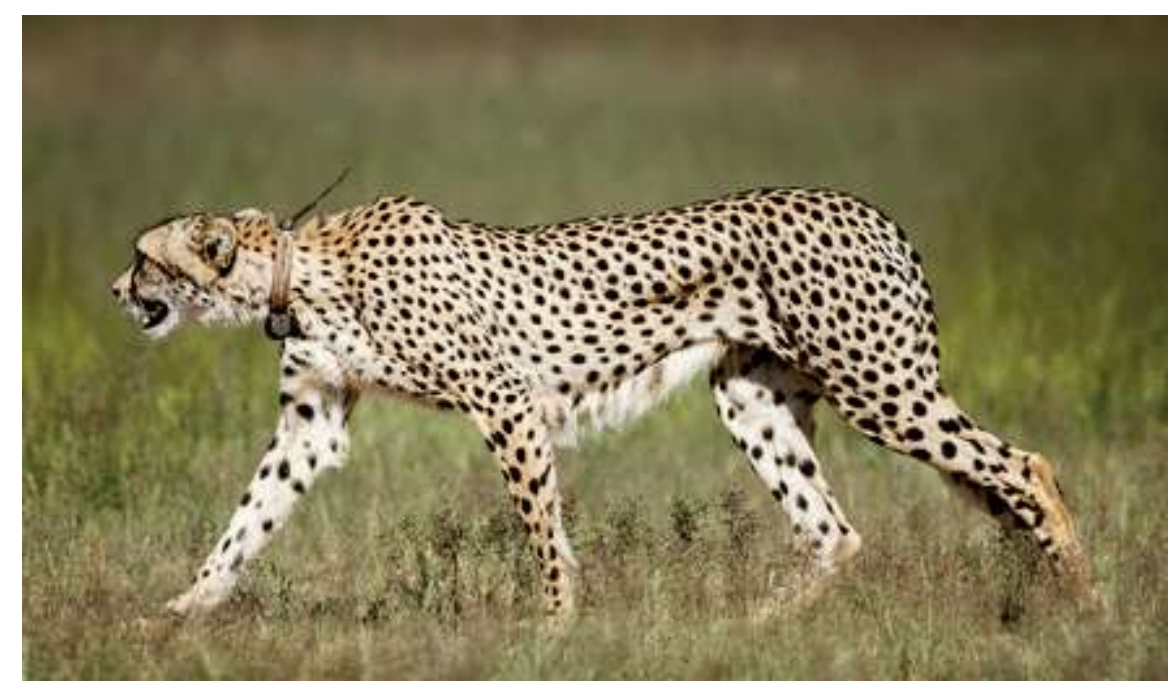

The effects of attaching data-collection tags to animals remain unknown.

PHOTO: (C) RICHARD DU TOIT/CORBIS

The 12 June Reviews by R. Kays et al. ("Terrestrial animal tracking as an eye on life and planet," p. 1222) and N. E. Hussey et al. ("Aquatic animal telemetry: A panoramic window into the underwater world," p. 1221) highlight some challenges to the future of terrestrial and aquatic telemetry studies, respectively, focusing on issues related to global collaboration and data sharing. Kays et al. also mention the need to continually improve animal-mounted sensors to minimize impacts of tags on animals. However, the gaps in our understanding of impacts associated with attaching instruments to animals are not given substantial consideration in either Review.

Potential impacts may be associated with capture/immobilization stress (1), increased drag (and its associated impacts on energy expenditure and locomotor performance) from external tags on aquatic and flying animals (2), behavioral modifications (3), and even environmental impacts such as biofouling (the accumulation of microorganisms or plants on wet surfaces)

(4). The requirement for more studies assessing tagging impacts has been recognized for some time [e.g., (5)]. Some recent papers reported impacts ranging from negligible $(6,7)$ to substantial $(8,9)$, but the paucity of such studies remains. In fact, in a review of papers reporting results from biologging deployments on free-ranging marine mammals (1965 to 2013; $n=620$ ), I only found 14 papers explicitly aimed to quantify potential impacts associated with instrument deployments (10). The knowledge contributions of telemetry studies are undeniable and auspicious, but adequately measuring and minimizing possible negative instrument effects remain important challenges and should receive increased research interest. 


\section{References}

1. A. M. M. Baylis et al., Mar. Mammal. Sci. 31, 322 (2015).

2. C. Tudorache et al., PLOS ONE 9, e112280 (2014).

3. J. M. van der Hoop et al., J. Exp. Biol. 217, 4229 (2014).

4. R. R. Reisinger et al., Polar Biol. 33, 561 (2010).

5. R. P. Wilson, C. R. McMahon, Front. Ecol. Environ. 4, 147 (2006).

6. R. R. Reisinger et al., PLOS ONE 9, e111835 (2014).

7. Y. Kim et al., Mar. Ornithol. 42, 63 (2014).

8. S. P. Vandenabeele et al., Mar. Ecol. Prog. Ser. 519, 239 (2015).

9. A. L. Rasiulis et al., J. Wildl. Manage. 78, 953 (2014).

10. T. McIntyre, Afr. J. Mar. Sci. 36, 409 (2014). 